\title{
Editorial
}

\section{Arterial Stiffness as a Cardiovascular Risk Factor in Stage 5D Chronic Kidney Disease Patients: An Age Affair}

\author{
Carmine Zoccali ${ }^{a}$ Francesca Mallamaci ${ }^{a, b}$ \\ ${ }^{a}$ CNR-IFC, Clinical Epidemiology and Pathophysiology of Hypertension and Renal Diseases, and bephrology, \\ Hypertension and Renal Transplantation Unit, Ospedali Riuniti, Reggio Calabria, Italy
}

In the general population, arterial stiffening is a strong risk factor for incident hypertension and cardiovascular (CV) events including fatal or nonfatal myocardial infarction, unstable angina, heart failure and ischemic or hemorrhagic stroke. Beyond and above hypertension, arterial stiffness per se has a relevant impact on $\mathrm{CV}$ disease because several treated hypertensives still show a high degree of arterial stiffness, a finding that may explain some of the residual CV risk that remains in well-controlled hypertension [1]. Widening in pulse pressure and high systolic pressure are recognized as robust markers of arterial stiffening. In this regard, it cannot be overemphasized that pulse pressure emerged as the strongest BP metric for the risk of death in a cohort of 37,069 patients across 752 hemodialysis facilities in the USA in a classical study published in the early years of the new millennium [2].

Although the damaging potential of arterial stiffening on the CV system has been unequivocally proven in the general population, gaps in our understanding of the clinical risks associated with arterial stiffening in some conditions and clinical settings remain. One of these conditions is stage $5 \mathrm{D}$ chronic kidney disease (CKD). Several studies in stages 3-5 CKD patients and in stage $5 \mathrm{D}$ CKD patients elucidated mechanisms responsible for arterial stiffening (depicted in fig. 1) and coherently associ-

\section{KARGER}

(c) 2016 S. Karger AG, Basel

E-Mail karger@karger.com

www.karger.com/ajn ated this alteration with surrogate end points like left ventricular hypertrophy or intima media thickening or endothelial dysfunction. Until now, there are only 3 reasonably large studies in stage 5D CKD patients investigating the relationship between a main parameter of vascular stiffening like pulse wave velocity (PWV) and mortality and CV events [3-5]. These studies were all limited by the small number of clinical events which ranged from 73 [3] to 143 [5] and from 36 to 48 for all-cause and CV death $[3,4]$, respectively, and the largest of these studies [5] did not report information on CV death. Furthermore, formal interaction analysis for the identification of factors that can modify the relationship between PWV and clinical outcomes was performed in none of those studies. This is an important lack of information in the stage 5D CKD population. As a matter of fact, in community level studies, women appear to be more susceptible to the harmful effect of pulsatile arterial load on diastolic function [6]. Furthermore, an established biomarker of arterial stiffness like pulse pressure amplification, but not arterial pressure, predict mortality in the elderly [7]. Whether older age modifies the link between PWV and clinical outcomes has not been specifically tested in the cohort studies performed in stage 5D CKD patients so far [3-5]. 


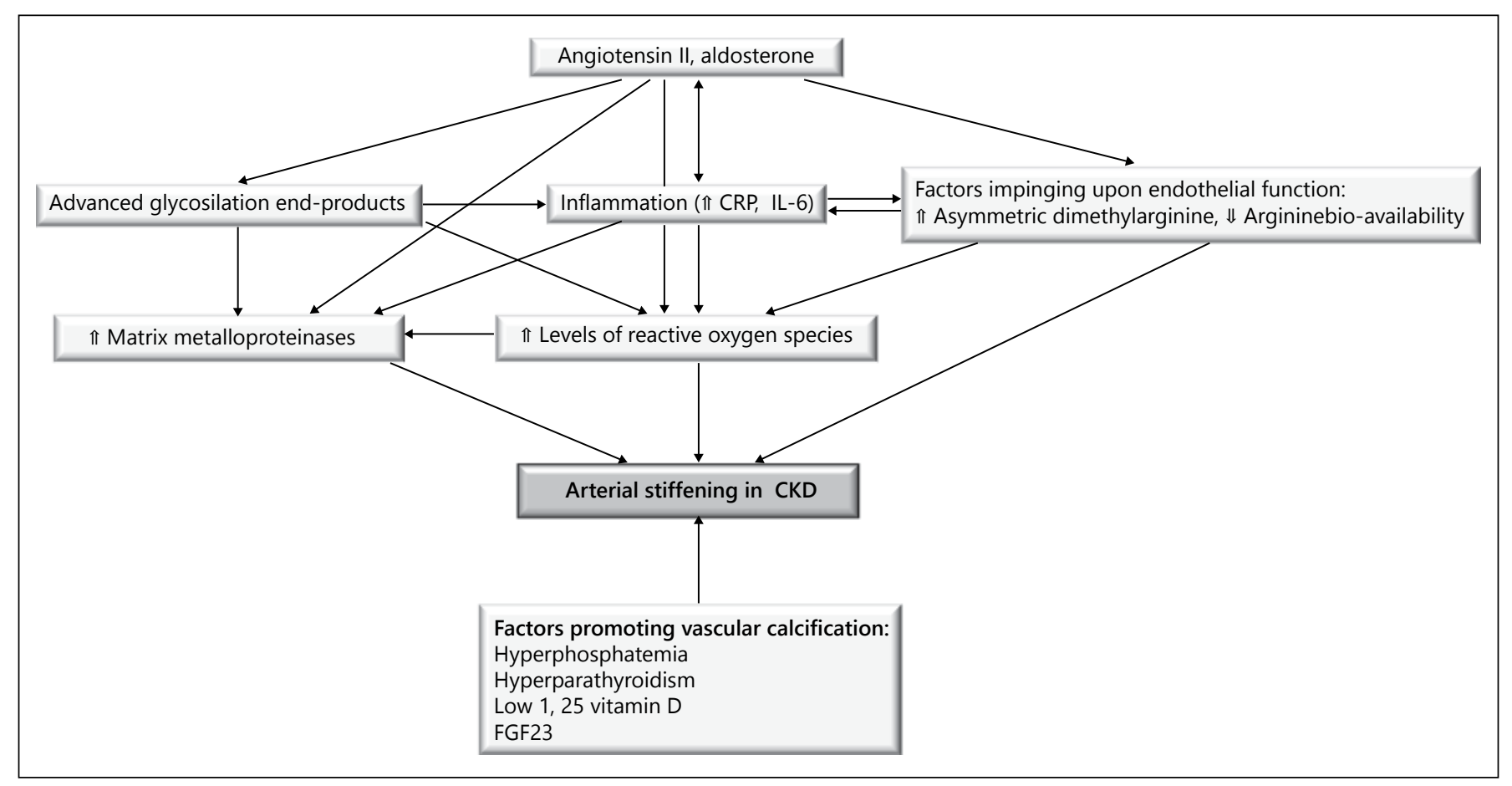

Fig. 1. Factors implicated in arterial stiffening in $\mathrm{CKD}$ and their interplay into the same process.

In a paper published in this issue of the Journal [8], Ferreira et al. [8], for the first time, test the hypothesis that age may be a crucial factor modifying the relationship between PWV and all-cause and CV mortality. The study, now extended to 278 patients, builds upon the seminal cohort described by Blacher et al. [3], in the first paper reporting the predictive power of PWV for all-cause and CV mortality in stage 5D CKD patients. This is a datarich, well-characterized cohort whereupon several important observational studies on CV risk have been performed over the last 20 years by the Manhes Hospital Research group headed by Gerard London. In this enlarged cohort, about one-thirds of patients had a PWV of $>12 \mathrm{~m} / \mathrm{s}$, which is a definitely high value of this parameter. After a median follow-up of about 6 years, $91 \mathrm{CV}$ deaths were registered. In this new analysis, a strong interaction emerges between age and PWV for the prediction of $\mathrm{CV}$ death. Herein, PWV predicts a very high risk indeed (HR 14 !) in patients younger than 60 years while the risk for the same outcome in those $>60$ years largely fails to attain statistical significance. Of note, in these novel analyses, PWV in patients $<60$ years improved the CV risk discrimination and risk reclassification in a model adjusting for established risk factors for $\mathrm{CV}$ disease in advanced $\mathrm{CKD}$. Yet, however strong the association be- tween PWV and CV death in the population $<60$ years, these observations cannot be immediately translated into prognostic recommendations. The effect modification by age on PWV for clinical outcomes has biological underpinnings, but from a statistical point of view interaction analyses, particularly when made in relatively small cohorts like the Manhes cohort, should be cautiously interpreted. This is so because testing for several interactions inflates the possibility of false positive findings. Only when confirmed in at least in one additional, sufficiently large, external cohort can the PWV-age interaction be proposed for prognostic purposes. Even though the stateof-the-art prognostic analyses like risk discrimination and risk reclassification have been applied, correctly so, Ferreira et al. [8] refrain themselves from suggesting that their findings are important for prognosis. Rather, they interpret their findings in an etiologic/therapeutic perspective concluding that PWV may serve to guide CV therapy in stage 5D CKD patients. Until now, no study has ever been performed to test a treatment policy guided by PWV in stage $5 \mathrm{D}$ CKD patients. Given the exceedingly high $\mathrm{HR}$ of this condition and the lack of benefit of several treatments tested in previous studies in the same population, such a trial appears to be of obvious clinical importance. If and when the trial will be done, the fresh 
findings by Ferreira et al. [8] will prove useful for defining the target population whereupon focusing the therapeutic intervention(s) aimed at modifying arterial rigidity. Indeed, if confirmed in future analyses in existing databases and in future observational studies, Ferreira et al. [8] data indicate that younger rather than older dialysis patients may be more likely to benefit from effective intervention(s) impinging upon arterial rigidity. Because of the paramount role of $\mathrm{CV}$ disease in the high mortality rate of dialysis patients, investigating the issue is not at all a trivial problem. Indeed, on a world scale, this population now exceeds 2.5 millions and it is expected to be 5 million by 2030 .

\section{Disclosure Statement}

The authors have no conflicts of interest to declare.

\section{References}

1 Niiranen TJ, Kalesan B, Hamburg NM, Benjamin EJ, Mitchell GF, Vasan RS: Relative contributions of arterial stiffness and hypertension to cardiovascular disease: the Framingham Heart Study. J Am Heart Assoc 2016; 5:e004271.

2 Klassen PS, Lowrie EG, Reddan DN, DeLong ER, Coladonato JA, Szczech LA, et al: Association between pulse pressure and mortality in patients undergoing maintenance hemodialysis. JAMA 2002;287:1548-1555.

3 Blacher J, Guerin AP, Pannier B, Marchais SJ, Safar ME, London GM: Impact of aortic stiffness on survival in end-stage renal disease. Circulation 1999;99:2434-2439.
4 Shoji T, Emoto M, Shinohara K, Kakiya R, Tsujimoto $\mathrm{Y}$, Kishimoto $\mathrm{H}$, et al: Diabetes mellitus, aortic stiffness, and cardiovascular mortality in end-stage renal disease. J Am Soc Nephrol 2001;12:2117-2124.

5 Fortier C, Mac-Way F, Desmeules S, Marquis K, De Serres SA, Lebel M, et al: Aortic-brachial stiffness mismatch and mortality in dialysis population. Hypertension 2015;65: 378-384.

6 Coutinho T, Borlaug BA, Pellikka PA, Turner ST, Kullo IJ: Sex differences in arterial stiffness and ventricular-arterial interactions. J Am Coll Cardiol 2013;61:96-103.
7 Benetos A, Gautier S, Labat C, Salvi P, Valbusa F, Marino F, et al: Mortality and cardiovascular events are best predicted by low central/peripheral pulse pressure amplification but not by high blood pressure levels in elderly nursing home subjects: the PARTAGE (Predictive Values of Blood Pressure and Arterial Stiffness in Institutionalized Very Aged Population) study. J Am Coll Cardiol 2012;60:1503-1511.

8 Ferreira JP, Girerd N, Pannier B, Rossignol P; London GM: High pulse wave velocity defines a very high cardiovascular risk cohort of dialysis patients under age 60 . Am J Nephrol 2016;45:72-81. 